\title{
Algunas explicaciones para el grado de penetración de la banda ancha
}

\author{
Juan Rubio Martín \\ Profesor de Economía Aplicada \\ Universidad Complutense de Madrid \\ César Sánchez Pérez \\ Profesor de Economía \\ UNAM (México)
}

\begin{abstract}
Resumen
Los últimos años han estado marcados, en los mercados de las telecomunicaciones, y en particular en la banda ancha, por el debate entre dos grandes políticas regulatorias y, en consonancia con esto, dos enfoques regulatorios en cuanto a modelos de competencia: uno con especial hincapié en la promoción de la competencia entre infraestructuras (modelo de competencia plena en redes y servicios) y otro con énfasis en la promoción de la competencia dentro de una misma infraestructura (modelo de competencia restringida a los servicios). En este contexto, el presente artículo muestra la evidencia empírica de los beneficios del primer modelo para el desarrollo y penetración de la banda ancha, utilizando como herramientas el análisis econométrico y el análisis envolvente de datos (DEA, Data Envelopment Analysis).

Palabras clave: competencia, regulación, telecomunicaciones, banda ancha, fibra óptica, eficiencia, DEA.
\end{abstract}

Clasificación JEL: K21, K23, L63, L96, O33, O52.

\section{Abstract}

In recent years telecommunication market, particulary the broanband market, have been marked by debate over the two important regulatory policies, and as such, two focus on competition models: one with special emphasis in promoting competition among infrastructures (full competition model in networks and services, and another with emphasis in promoting competition within the same infrastructure (competition model restricted to services). It is within this context that our article presents empirical evidence on the benefits of the first model for the development and penetration of the broadband, using econometric analysis, as well as DEA (data envelopment analysis), as tools.

Keywords: competition, regulation, telecommunications, broadband, fibre optic, efficiency, DEA.

JEL classification: K21, K23, L63, L96, O33, O52.

\section{Introducción}

En 1998 se produjo la liberalización total (de servicios e infraestructuras) de las telecomunicaciones en el seno de la Unión Europea. El objetivo prioritario del nuevo marco era la construcción de un modelo de competencia sostenible, es decir, un modelo que propiciara el desarrollo de alternativas de red a las del operador histórico. 
La realidad de lo acontecido ha sido, al menos en varios países, diferente a lo que se había previsto, al haberse puesto más énfasis en la apertura del bucle del operador histórico, la reventa forzosa y la reducción de los precios que en el desarrollo del modelo de competencia sostenible antes señalado. Una competencia entre operadores con infraestructuras propias y tecnologías de acceso distintas, para así lograr un mayor desarrollo de la banda ancha, por tanto, entre agentes con capacidad autónoma e incentivos para la inversión e innovación.

La influencia de esta variable, «competencia entre tecnologías» o «competencia en redes», está siendo aún más relevante en varios países que las reducciones de precios en el desarrollo y expansión del mercado de Banda Ancha (en adelante BA), tal y como se muestra en el análisis econométrico y análisis DEA que se realiza en este artículo. Esta competencia no significa una «duplicación» de infraestructuras (zanjas, emplazamientos, postes, entre otros), sino que es compatible con el uso compartido de la obra civil existente, algo previsto desde el inicio de la liberalización y contemplado en la normativa vigente ${ }^{1}$. El coste de dicha obra civil supone la mayor parte de la inversión ${ }^{2}$, por lo que el uso compartido hace que disminuya considerablemente. En definitiva, la competencia entre redes (y estamos hablando de pocas redes) coexiste, en muchas ocasiones, con el uso compartido de infraestructuras, haciendo aquélla eficiente ya que los beneficios de la existencia de competencia compensan sobradamente el coste incurrido en la construcción de la red.

El modelo opuesto es aquel en el que operadores sin red ofrecen servicios sobre la red del operador histórico, a precio mayorista continuamente regulado; es decir, un modelo de «competencia regulada» y restringida a los servicios.

Este trabajo se organiza de la siguiente forma: primero se realiza un breve resumen de los antecedentes del estudio de la penetración de la banda ancha. Posteriormente se examina la situación de las diferentes tecnologías que soportan la BA en los países de la OCDE, y la evolución y convergencia de la BA en estos países, haciendo hincapié en el caso de España. Segundo, se mostrará cómo la elevación de la participación de tecnologías alternativas, como el cable, aumentan la competencia y el grado de penetración de BA. Se propone, además, en esta sección una forma de medir la competencia entre tecnologías. En tercer lugar, en la investigación empírica propiamente dicha, se realiza un estudio econométrico con el objetivo de mostrar que la competencia es un factor significativo en la explicación de la BA para el conjunto de países de la OCDE. Justificado esto, se procede a realizar un análisis no paramétrico DEA con resultados para cada uno de los países de la OCDE a fin de indagar la res-

1 Por ejemplo, artículo 12 Directiva Marco 2002/21/CE y Recomendación de la Comisión sobre el acceso regulado a las Redes de Acceso de Nueva Generación (20/9/2010, puntos 12 a 17, entre otros).

${ }^{2}$ Según estimaciones asumidas por reguladores, consultoras, etc., en el caso de las telecomunicaciones, hasta el 80 por 100 del coste de crear una red es obra civil, es decir, zanjas en calles y aceras, tubos, permisos de obra, etc. (construidas en época de monopolio). Véanse, por ejemplo, CMT, Resolución de 8/5/2008 sobre medidas cautelares en relación con la definición y análisis del mercado de acceso al por mayor a infraestructura de red (RE-2008-5-8-1-1), así como la Nota Explicativa de la Comisión Europea a la Recomendación de Mercados, p.17, SEC (2007) 1483 final. 
puesta de la BA a factores como: la competencia, los precios, el poder de compra y la BA en los negocios. Por último, se formulan algunas conclusiones y sugerencias.

\section{Antecedentes}

\subsection{Algunos trabajos sobre el estudio de la BA}

Desde el comienzo de la liberalización puede decirse que han existido dos posiciones contrarias en cuanto a políticas de desarrollo de la banda ancha. Una, apoyada en el acceso regulado a las redes de los operadores históricos y basada en la creencia de que el bucle local era muy difícil de replicar, y otra basada en la construcción de nuevas redes por operadores alternativos. El desarrollo en algunos países de verdaderas alternativas de red, con cuotas de mercado importantes, ha ido inclinando el debate a favor del desarrollo de la banda ancha vía competencia entre redes, sobre todo si la evidencia ha mostrado que así se aumenta en mayor medida la penetración.

La Comisión Europea (2004) ya indicaba que los mercados de banda ancha competitivos favorecen un crecimiento más rápido, y que existe una correlación positiva entre la competencia entre redes y el crecimiento de los índices de penetración.

Distaso et al., (2006) estudiaron varios países europeos y encontraron evidencia empírica de que una mayor competencia entre distintas tecnologías conduce a una mayor expansión de la banda ancha. Mostraron también que la competencia dentro de una misma tecnología (intra-tecnología) y la competencia a través de la desagregación del bucle no juegan un papel significativo en el desarrollo de la banda ancha.

Höffler (2007), por su parte, compara Europa con Estados Unidos y encuentra un porcentaje de hogares pasados por cable más alto en este último país y, por tanto, una competencia en banda ancha muy intensa, lo que se refleja en un impacto positivo y significativo en el nivel de penetración de la misma en EE.UU. en relación con Europa. No obstante, el dato más reciente que analiza los países europeos se refiere a principios de 2004. Se puede apreciar en el trabajo cómo en Europa, cuanto más alta es la penetración del cable en cada país, más alto es el nivel de penetración de banda ancha (relación de U invertida), aunque en términos de ganancia de excedente total no llega a conclusiones definitivas.

Cabe mencionar también el análisis empírico llevado a cabo por la consultora LECG (2007), que estimó la potencial pérdida de inversión debido a la intensa regulación del acceso al bucle en 10.000 millones de euros en los próximos diez años. El estudio empleaba técnicas econométricas para evaluar el impacto de la regulación del precio del bucle sobre la inversión en redes de acceso, utilizando datos del período 2002 a 2006. La conclusión es que una reducción del 10 por 100 en el precio del bucle causa un 18 por 100 de descenso en la cuota de mercado de las infraestructuras alternativas, en número de clientes, y una reducción agregada en el volumen de inversiones próxima a los 10.000 millones de euros. La recomendación principal del estudio es que los gobiernos y reguladores deberían considerar 
cuidadosamente el enorme coste de la regulación del acceso en términos de debilitar la inversión y la competencia de redes alternativas.

Friederiszick et al., (2008), con datos de 180 operadores en 25 países europeos a lo largo de diez años, han concluido que una fuerte regulación del acceso desincentiva a los entrantes de invertir en redes propias, aunque a corto plazo se produzca un descenso de precios. Asimismo, Wallsten y Hausladen (2009), con 27 países europeos en el período 2002-2007, encuentran una correlación negativa entre el número de bucles desagregados y las conexiones de fibra. Además, en cuanto a la regulación en EEUU, Hazlett (2005) muestra que la eliminación de la regulación de precios mayoristas es lo que lleva al desarrollo de la competencia en redes.

En lo que respecta a las Redes de Nueva Generación, y a pesar de que el despliegue es todavía muy limitado, sí que hay ya evidencias de que los países con mayor competencia entre infraestructuras (EE.UU., Suecia, Suiza, Corea, Japón) son los más avanzados ${ }^{3}$. Así, en Estados Unidos, donde existe una intensa competencia entre los incumbentes y operadores de televisión por cable, estos últimos han realizado en los últimos años fuertes inversiones sobre sus redes para proporcionar servicios IP avanzados, compitiendo por tanto en la oferta de acceso en banda ancha. Con esta competencia, la FCC, regulador de EE.UU., no ve necesario emprender acciones regulatorias en el acceso (en forma de servicios mayoristas «bitstream»o bucles desagregados, al modo de Europa).

Los análisis del despliegue de fibra óptica en el acceso muestran que éste es más rápido en países donde la televisión por cable posee una cuota de banda ancha elevada $^{4}$. Eisenhach (2008) explica cómo en EEUU y Canadá la competencia entre operadores de cable y de telefonía está generando volúmenes de inversión en infraestructuras avanzadas superiores a los europeos desde hace ocho años. Sin perjuicio de ello, en algunos países (caso de Japón) los gobiernos han podido en algún momento conceder subsidios a empresas en competencia, pero destinados a que las nuevas infraestructuras lleguen a zonas no rentables.

Sobre otras áreas geográficas menos desarrolladas, Montoya (2007), ha estudiado el efecto tanto de la independencia de las autoridades reguladoras como de la competencia inter-tecnologías en la penetración de la banda ancha de doce países de América Latina y el Caribe, incluyendo el índice de Herfindahl-Hirschman de tec-

\footnotetext{
${ }^{3}$ Si se mide a través de la penetración de fibra óptica, ver UIMP (2009). Asimismo, IDATE (2010) señala a Japón como el líder en abonados con fibra óptica (FTTH/B, Fiber to the Home/Fiber to the Building), mientras que en EE.UU. se alcanza en algunas zonas una penetración del 28.5 por 100, gracias a las enormes inversiones de los grandes operadores, sobre todo Verizon. En Europa, es Suecia, con gran diferencia sobre los demás, el líder en este campo. En Corea, uno de los líderes mundiales, existe una intensa competencia entre los operadores históricos y los alternativos. Así, según IDATE, KT tiene 3.5 millones de abonados de fibra óptica, pero operadores nacidos hace pocos años, como $L G$ Powercom, sobrepasan los 1.5 millones (datos a junio de 2009). Esto sin perjuicio de que a principios de la década pasada el gobierno coreano estableciera «políticas nacionales» para estimular el desarrollo de infraestructuras y, al mismo tiempo que se liberalizaba y privatizaba el mercado, existieran medidas de apoyo a la innovación tecnológica (CHOONGOK, 2004).

4 Véase también KATZ (2008): La competencia entre plataformas: teoría y resultados, Madrid, Enter.
} 
nologías (IHH, una sumatoria de cuadrados de participaciones), que mediría el grado de concentración tecnológica en el mercado (DSL, cable, banda ancha inalámbrica). Dicho autor concluye señalando que una menor concentración tecnológica lleva, de forma significativa, a una mayor penetración de banda ancha ${ }^{5}$. En cuanto al impacto de la independencia regulatoria, obviamente, los resultados indican que la mayor independencia impulsa la penetración de banda ancha.

Finalmente, destacan los trabajos econométricos llevados a cabo en los últimos años por el Phoenix Center, que establecen relaciones del crecimiento de la banda ancha con variables tales como la educación, la edad, la desigualdad en la distribución de la renta, la densidad de población y otros factores. Estos trabajos están centrados fundamentalmente en EE.UU.

En conclusión, parece haber un consenso académico mayor a favor de la adopción de un modelo de competencia en redes ${ }^{6}$ si se quiere lograr mejores resultados en términos de desarrollo y penetración de servicios de banda ancha. Obviamente, a más competencia, más penetración y más rápido crecimiento de ésta; y los operadores alternativos de ADSL sin red de acceso propia contribuirán, qué duda cabe, a la pérdida de cuota del operador histórico, pero solo a corto plazo, pues se trata de una competencia no sostenible a medio-largo plazo $^{7}$, es decir, centrada en el día a día sin un marco claro y estable.

En países con más participación de cable, alternativa principal de la tecnología DSL del operador histórico, la penetración global de banda ancha es más alta y crece más deprisa. Ello sin perjuicio de que en determinadas zonas rurales, donde al mercado no le resulte rentable invertir, puedan ser necesarias medidas de ayuda siempre que éstas sean neutras y con acceso abierto y no discriminatorio (Comisión Europea, 2009). Una cuestión es que las autoridades nacionales elaboren planes de despliegue de red con objetivos de cobertura y velocidad y otra que los estados intervengan directamente en el mercado ${ }^{8}$.

5 En línea, por tanto, con los resultados de DISTASO et al., (2006) y HÖFFLER (2007). Este último, en su artículo «Costs and Benefits from Infrastructure Competition. Estimating Welfare Effects from Broadband Access Competition» presenta el conocido gráfico en forma de «U invertida» entre la cuota de mercado del cable y el grado de penetración de la banda ancha; es decir, en cuotas del orden del 40 por 100 en cable se llega a dar los mayores niveles de penetración, como es el caso de Bélgica, Dinamarca, Suecia y Holanda. A nivel de teoría económica, el llamado comportamiento de la «U invertida ha sido tratado por AGHION (2005), citado más adelante, para establecer la relación entre el número de empresas y el ritmo de innovación. Anteriormente el fenómeno ya fue identificado por SCHERER (1967).

${ }^{6}$ Esto no significa que no haya también posturas a favor de la regulación de la infraestructura principal para incentivar la inversión. Véase CAMBINI y JIANG (2009), entre otros.

7 Esto es debido a que buena parte de las empresas de este tipo han sido efímeras. Más adelante, como se ha visto en el caso de España, el operador ex monopolista vuelve a recuperar la cuota perdida, que contrasta con la pérdida de cuota experimentada por los principales alternativos y la desaparición de los más pequeños. Así, en 2009 las líneas de banda ancha de Telefónica representaban el 54,9 por 100 del total, frente al 53,7 por 100 de 2005 (CMT, 2010, p. 315).

${ }^{8}$ La intervención estatal puede ir algo más allá, como en los casos de Australia y Nueva Zelanda (GIVEN, 2010), donde los gobiernos forman empresas público-privadas para redes de fibra óptica, pero únicamente a escala mayorista y con acceso abierto a todos los operadores, es decir, sin ofrecer servicios finales. En Australia, «retando» al operador dominante. 
El mecanismo de la escalera de inversión, que empezó a conocerse después de la liberalización total europea de 1998, y ante su fracaso ${ }^{9}$, está llevando a una competencia restringida a los servicios, ya que no culmina al final en una competencia sostenible basada en infraestructuras ${ }^{10}$.

\subsection{Situación de la banda ancha en la OCDE}

Las diferentes tecnologías en el conjunto de países de la OCDE muestran patrones bien definidos. La plataforma DSL es la tecnología dominante. El Gráfico 1 presenta esta información a junio de 2010, alcanzando un 58,4 por 100. En un año comparable en la desagregación de tecnologías, en 2006 , tenía ya un 62,4 por 100 . El cable muestra un leve avance: para 2010 tiene una cuota del 29,2 por 100, mientras que en 2006 era del 29,0 por 100. La fibra + LAN, en cambio, avanza hasta un 11,8 por 100 para 2010, cuando en 2006 representaba sólo el 6,46 por 100.

FUENTE: OCDE.

\section{GRÁFICO 1}

USO DE TECNOLOGÍAS. PAÍSES DE LA OCDE, 2010

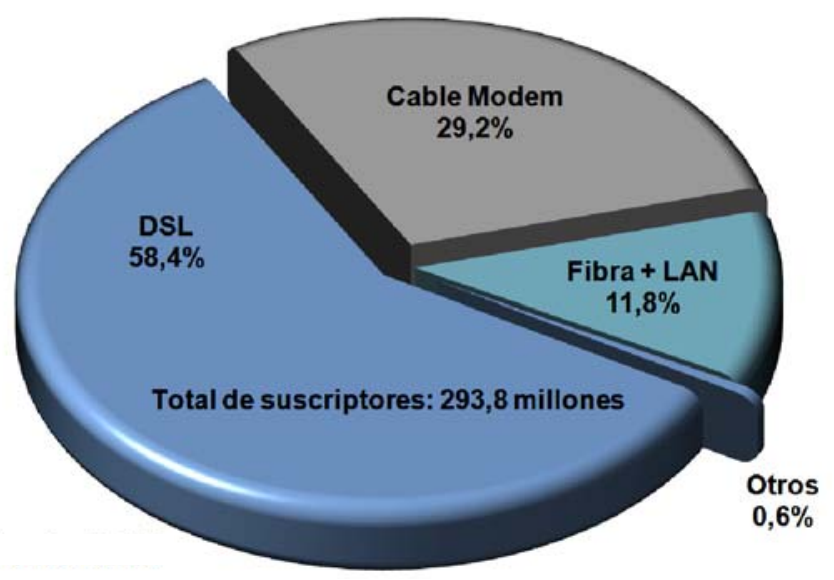

${ }^{9}$ En parte debido a la no aplicación de instrumentos regulatorios tal y como se preveía inicialmente, a saber, precios de acceso a la red del incumbente crecientes y cláusulas de caducidad del mecanismo (BOURREAU et al., 2010). En el artículo de Bourreau se señala también el freno a la innovación en tecnología de red que supone la implantación de la «escalera de inversión».

10 Los entrantes se quedan en un peldaño intermedio. Cuando se desagregan los bucles, han de pagar tanto un precio de alta como una cuota mensual por cada línea, teóricamente orientada al coste, pero en realidad cara si se tiene en cuenta las prácticas de estrechamiento de márgenes de los últimos años, donde los monopolistas han mantenido e incluso recuperado cuota de mercado (caso de España). 
En definitiva, existen tres principales plataformas desde las que se pueden ofrecer servicios de banda ancha: Digital Suscriber Line (DSL), característica de las redes mejoradas de los antiguos monopolios; cable modem, redes más modernas con origen en la TV por cable; y Fibra más otras minoritarias. En Europa domina claramente la tecnología DSL, por lo que, dado que en varios países se ha adoptado el modelo de la escalera de inversión en lugar de favorecer desde el principio el despliegue de redes alternativas, se trataría de incentivar a potenciales operadores verdaderamente alternativos (con red de acceso propia) a dar el salto en la escalera que les llevase a desplegar sus propias infraestructuras de acceso a los clientes finales. Esto habría de hacerse con la ayuda de las medidas ya previstas en la legislación de uso compartido de canalizaciones, registros, etc.

De esta forma se evitaría un retroceso en la situación competitiva de los mercados de banda ancha, ya que se prolongaría la situación actual de competencia regulada y no sostenible por sí misma, que podría ser irreversible, cuando los operadores dominantes lanzaran sus ofertas comerciales desde las infraestructuras de acceso de nueva generación hasta los hogares. No sería posible la libre acción económica de los operadores alternativos, y se les condenaría a ir siempre a remolque de los planes, ritmo y alcance del operador correspondiente ${ }^{11}$.

El Cuadro 1 resume las tecnologías en la OCDE a diciembre de 2009. Si bien ya se ha publicado la información a junio de 2010, ésta no representa cambios significativos y no modifica las conclusiones del análisis presente. Puede observarse en 2010 las diferencias entre grupos de países: la media ponderada de países desarrollados es de 27,7 por 100 , mientras que el otro grupo tiene una media de 11,3 por 100. Esta diferencia se veía ya en 2009. Destacan además las ventajas del grupo desarrollado; en éstos la media en 2009 en DSL es del doble; sin embargo, la media en cable es cuatro veces más y ocho en Fibra.

11 Por un lado, por tener que retroceder los alternativos hacia posiciones más de «reventa», haciéndoles aún más dependientes de lo que hiciera el dominante; y por otro, por la probable situación de competencia exclusivamente en precios con un claro ganador como ha mostrado la experiencia: aquel que posea el músculo financiero suficiente, es decir, el dominante. Éste podría, de nuevo, proceder a ajustes de plantillas y otras medidas para consolidar su situación, como ha venido ocurriendo en España y otros países, y recuperar buena parte de las cuotas de mercado que inicialmente perdió con la liberalización. 
CUADRO 1

INDICADORES DE BANDA ANCHA.

OCDE, 2009 y 2010

\begin{tabular}{|c|c|c|c|c|c|c|c|c|c|}
\hline \multirow{2}{*}{\begin{tabular}{|c|} 
Año \\
Rank
\end{tabular}} & \multicolumn{8}{|c|}{2009} & \multirow{2}{*}{$\begin{array}{c}2010 \\
\text { BA } \\
\text { por } \\
100 \\
\text { hab. }\end{array}$} \\
\hline & País & DSL & Cable & $\begin{array}{c}\text { Fibra } \\
\text { y LAN }\end{array}$ & Otros & $\begin{array}{c}\text { Cable } \\
\text { +Fibra } \\
\text { +LAN } \\
\text { +otros }\end{array}$ & $\begin{array}{c}\text { BA } \\
\text { por } \\
100 \\
\text { hab. }\end{array}$ & $\begin{array}{c}\text { Total } \\
\text { clientes }\end{array}$ & \\
\hline 1 & Holanda & 22,1 & 14,2 & 0,8 & 0 & 15,0 & 37,1 & 6131000 & 37,8 \\
\hline 2 & Dinamarca & 22,4 & 10,1 & 4,2 & 0,3 & 14,6 & 37,1 & 2046000 & 37,3 \\
\hline 3 & Suiza & 25,1 & 10 & 0,3 & 0,3 & 10,6 & 35,6 & 2780050 & 37,1 \\
\hline 4 & Noruega & 20,9 & 8,6 & 4,3 & 0,1 & 13,0 & 33,9 & 1637500 & 34,2 \\
\hline 5 & Corea & 6,6 & 10,6 & 16,4 & 0 & 27,0 & 33,5 & 16347716 & 34,4 \\
\hline 6 & Islandia & 30,7 & 0 & 2,2 & 0 & 2,2 & 32,8 & 104770 & 33,3 \\
\hline 7 & Suecia & 17,8 & 6,2 & 7,4 & 1 & 14,6 & 32,4 & 3022379 & 31,8 \\
\hline 8 & Luxemburgo & 26,5 & 5,2 & 0,1 & 0,1 & 5,4 & 31,9 & 158548 & 34,1 \\
\hline 9 & Francia & 28,7 & 1,6 & 0,1 & 0 & 1,7 & 30,4 & 19582000 & 31,4 \\
\hline 10 & Alemania & 27,4 & 2,8 & 0,2 & 0 & 3,0 & 30,3 & 24843700 & 31,3 \\
\hline 11 & Canadá & 13,2 & 16,4 & 0 & 0 & 16,4 & 29,6 & 9980000 & 30,1 \\
\hline 12 & Reino Unido & 23,3 & 6,2 & 0 & 0 & 6,2 & 29,5 & 18213290 & 30,5 \\
\hline 13 & Bélgica & 16,5 & 12,3 & 0,2 & 0,1 & 12,6 & 29 & 3133881 & 30,0 \\
\hline 14 & Finlandia & 22,2 & 4,2 & 0,2 & 0,1 & 4,5 & 26,7 & 1427200 & 26,4 \\
\hline 15 & Estados Unidos & 10,7 & 14,1 & 1,3 & 0,3 & 15,7 & 26,4 & 81146225 & 27,1 \\
\hline 16 & Japón & 7,9 & 3,4 & 13,5 & 0 & 16,9 & 24,8 & 31630781 & 26,3 \\
\hline 17 & Australia & 19 & 4,2 & 0 & 0 & 4,2 & 23,3 & 5133000 & 23,4 \\
\hline 18 & Nueva Zelanda & 21,7 & 1,5 & 0 & 0 & 1,5 & 23,2 & 992383 & 24,5 \\
\hline 19 & Austria & 15,1 & 6,8 & 0,1 & 0,1 & 7,0 & 22,1 & 1844848 & 23,0 \\
\hline 20 & España & 17,1 & 4 & 0,1 & 0 & 4,1 & 21,2 & 9786578 & 22,2 \\
\hline 21 & Italia & 19,9 & 0 & 0,6 & 0 & 0,6 & 20,5 & 12338502 & 21,3 \\
\hline 22 & Irlanda & 16 & 3,4 & 0,1 & 0 & 3,5 & 19,5 & 870562 & 20,3 \\
\hline 23 & Portugal & 10,4 & 7,2 & 0,3 & 0 & 7,5 & 17,9 & 1902273 & 18,9 \\
\hline 24 & Hungría & 8,2 & 8,4 & 1,1 & 0,1 & 9,6 & 17,8 & 1785046 & 18,7 \\
\hline 25 & Grecia & 17 & 0 & 0 & 0 & 0,0 & 17 & 1918630 & 18,7 \\
\hline 26 & R. Checa & 7,4 & 4,2 & 1,3 & 0 & 5,5 & 12,9 & 1354986 & 13,7 \\
\hline 27 & Polonia & 8,1 & 3,9 & 0,1 & 0 & 4,0 & 12,1 & 4620000 & 13,1 \\
\hline 28 & República Eslovaca & 6,8 & 1,5 & 3,3 & 0 & 4,8 & 11,6 & 627722 & 12,0 \\
\hline 29 & Chile & 9,6 & 0 & 0 & 0 & 0,0 & 9,6 & 1630943 & 10,2 \\
\hline 30 & México & 6,8 & 2 & 0 & 0,5 & 2,5 & 9,2 & 9921407 & 10,1 \\
\hline 31 & Turquía & 8,6 & 0,2 & 0,1 & 0,1 & 0,4 & 9 & 6446374 & 9,4 \\
\hline \multicolumn{7}{|c|}{ Media ponderada (con población estimada a 2010) } & \multicolumn{3}{|c|}{ Suma } \\
\hline \multirow{3}{*}{\multicolumn{2}{|c|}{$\begin{array}{l}\text { OCDE } \\
\text { Países 1-23 } \\
\text { Países 24-31 }\end{array}$}} & 13,9 & 6,7 & \multirow{3}{*}{\multicolumn{2}{|c|}{$\begin{array}{l}\mathbf{2 , 7} \\
\mathbf{3 , 4} \\
\mathbf{0 , 4}\end{array}$}} & - & 23,3 & 283358294 & 24,3 \\
\hline & & 15,9 & 8,0 & & & - & 27,0 & - & 27,7 \\
\hline & & $\mathbf{8 , 0}$ & 2,0 & & & - & 10,5 & - & 11,3 \\
\hline
\end{tabular}

FUENTE: OCDE. Los datos de las diferentes tecnologías se muestran a diciembre de 2009, pero se exponen los datos de penetración de Banda Ancha Total a junio de 2010. Las medias ponderadas excluyen a Chile.

Se puede observar que los primeros dieciséis países concentran el 78,4 por 100 del total de suscriptores; por otro lado, los países con mejor ranking presentan una mayor diversificación en las plataformas tecnológicas; esto puede verse en la columna que suma a todas la tecnologías alternativas a DSL. De este modo, en el grupo de la OCDE los primeros dieciséis puestos del ranking tienen casi el 80 por 100 de los suscriptores y están al mismo tiempo bajo una mayor diversificación tecnológica. Ahora se observará la evolución de los países líderes de BA y de España. La evolución de Dinamarca, Holanda, Suiza y Noruega se ha acelerado considerablemente, 
hasta superar el 35 por 100 en 2009-2010, emulando incluso, desde 2006, al líder mundial durante muchos años, Corea (véase Gráfico 2).

\section{GRÁFICO 2}

\section{BANDA ANCHA, PAÍSES LIIDERES OCDE Y ESPAÑA, 2001-2010}

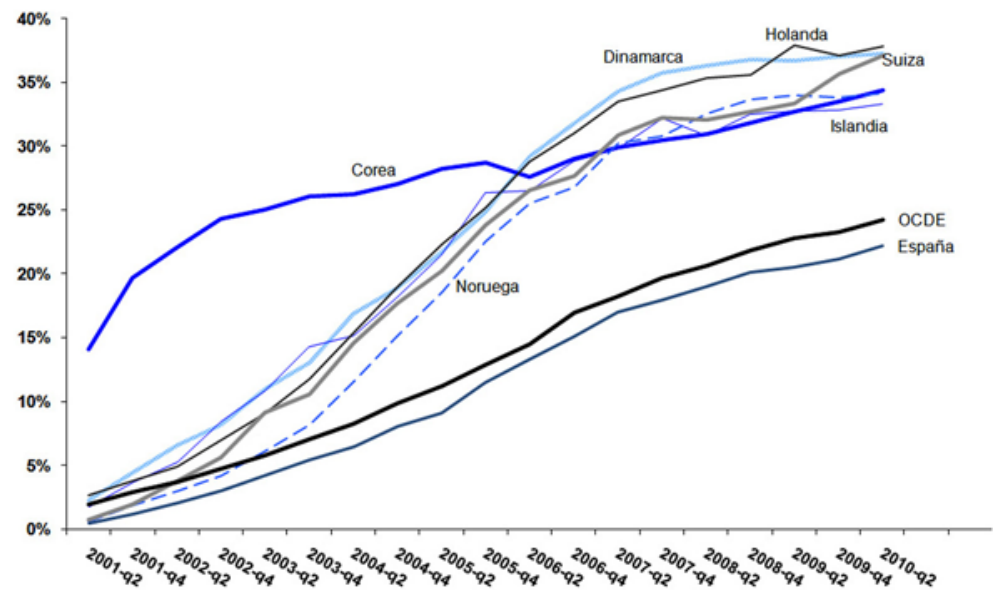

FUENTE: OCDE. Los datos provienen de información semestral desde 2001-2010.

¿Qué es lo que tienen en común estos países? El hecho de que no solo son líderes en penetración de banda ancha sino que al mismo tiempo son, en general, líderes en penetración de infraestructuras alternativas (cable, que es la principal alternativa desde la liberalización, y fibra/LAN más recientemente). De forma que, en estos países, los operadores que en su día apostaron por construir sus propias redes de acceso han tenido éxito. En cambio los países que están a la cola en penetración de banda ancha son precisamente los que tienen, en general, una menor penetración de infraestructuras alternativas, es decir, aquellos países cuya regulación estuvo centrada en el acceso regulado a las redes de los operadores históricos (véase Cuadro 1).

Como también puede observarse en el gráfico, existen dos períodos diferenciados de crecimiento. Para la OCDE, la tasa media anual de crecimiento del primer semestre de 2001 al primero de 2006 es de 21,9 por 100, sin embargo, para el segundo periodo del primer semestre del 2006 al primero de 2010 es de sólo 6,6 por 100. Para el primer periodo, Holanda y Dinamarca tuvieron un 26,7 por 100 y 28,9 por 100 respectivamente, pero de la misma forma, para el segundo periodo sólo creció su BA en un 3,5 por 100 y 3,1 por 100. España, en cambio, para el primer período tuvo un espectacular 40 por 100 de crecimiento, pero éste declinó al 6,6 por 100 de la OCDE.

\subsection{Evolución y convergencia en Banda Ancha en la OCDE}

La evolución y dispersión de la banda ancha en los países de la OCDE ha sido muy desigual, y lo mismo puede decirse para España en ese grupo. Este análisis es 
importante, porque permite indagar sobre la posible convergencia o divergencia del grupo y la situación de España en particular. Un histograma permite visualizar estos objetivos. Sin embargo, un suavizado mayor puede realizarse mediante una función de distribución de probabilidad (FDP), posibilitando, además, el cálculo del valor medio, de modas (agrupamientos), del coeficiente de variación (CV), etcétera. Este último dato manifiesta precisamente el proceso de convergencia.

El Gráfico 3 muestra las FDP para 2003 y 2010, donde el avance de la media de banda ancha es de 6,94 por 100 y 24,06 por 100 respectivamente (la media ponderada de 2009 se situó en un 23,3 por 100). Existe un proceso de convergencia en la OCDE, ya que para los mismos años, el CV se reduce de 0,783 a 0,344 (para 2009 fue de 0.354 ). Resumiendo, existe un proceso de convergencia indiscutible. Sin embargo, resalta la evidente consolidación de la bimodalidad en 2010. La situación de España en 2003 estaba por debajo de la media de la OCDE y del grupo modal de países europeos. Esta brecha ha aumentado ligeramente en 2010. De este modo, la brecha que separaba España de la media de la OCDE es de 1,55 en 2003 y de 1,87 en 2010. La brecha es aún más amplia si toma en cuenta la moda de los países líderes en la FDP.

\section{GRÁFICO 3}

FUNCIONES DE DENSIDAD DE PROBABILIDAD

(2003-2010)

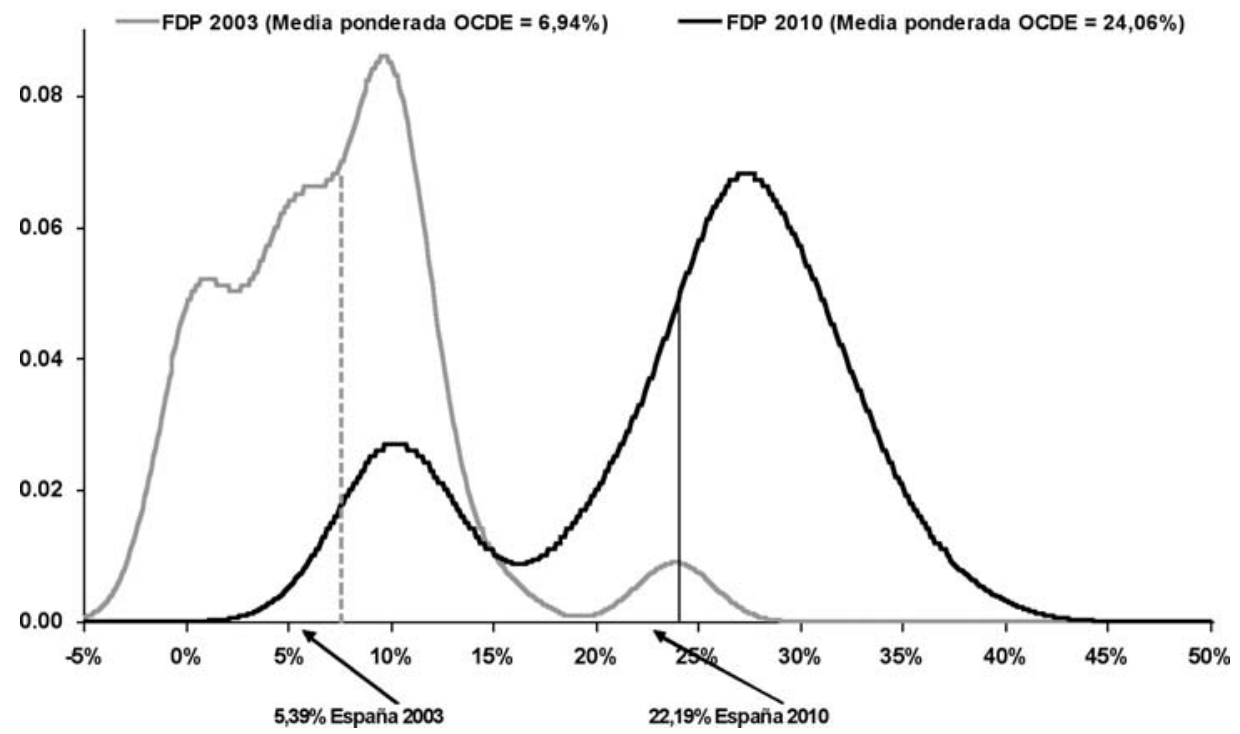

FUENTE: OCDE. Los datos provienen de la información proporcionada semestralmente

Queda claro que el reto para España está en converger dentro del grupo de países líderes, cuya moda en 2010 se establece en 27,4 por 100 (en 2009 es 26,6 por 100). La formación de estos dos grupos se tomará en cuenta en la modelación econométrica posterior. 


\section{La Banda Ancha y algunas formas de medir la competencia}

\subsection{Banda Ancha y participación del cable. $L a$ «U invertida»}

En este apartado se relacionará la BA y la competencia entre tecnologías mediante un recurso sencillo, la participación de cable (alternativa principal del xDSL de los operadores históricos). El significado de la «U invertida» es muy simple: a través de un ajuste cuadrático por Mínimos Cuadrados Ordinarios (MCO), la BA tiene una respuesta lineal ante un incremento de la participación del cable, pero en la medida en que este sobrepasa su participación óptima y monopoliza, pasa a tener un efecto negativo sobre BA. Los datos del Gráfico 4 (para 21 países seleccionados) se ajustan a una «U invertida ${ }^{12}$, siendo los promedios del período. De este modo se eliminan algunos movimientos atípicos interanuales principalmente en los años iniciales.

\section{GRÁFICO 4}

RELACIÓN ENTRE PORCENTAJE PENETRACIÓN DEL CABLE Y PORCENTAJE PENETRACIÓN DE BANDA ANCHA PROMEDIOS (2003-2009)

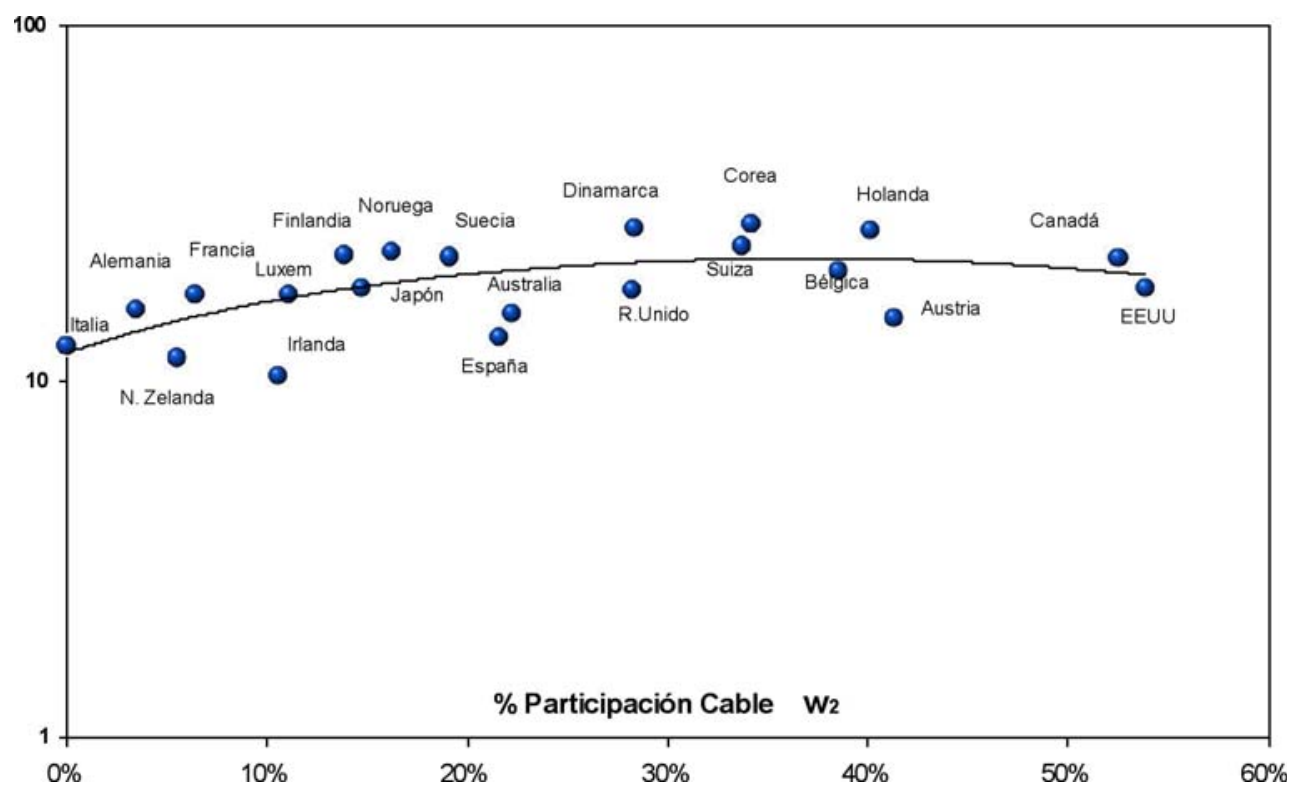

FUENTE: Elaboración propia con datos de la OCDE.

Una lectura más detenida nos indica que, al disponer de series de datos de tres tipos de tecnologías: DSL, cable y otras (fibra/LAN), el máximo del ajuste proporcionado por MCO se encuentra alrededor del 33 por 100 de la participación del

12 En línea con el trabajo de HÖFFLER (2007). 
cable (es decir, una participación igualitaria del cable) ${ }^{13}$. En este nivel se encuentra un país considerado paradigmático, Corea (de 2003 a 2009 el cable ha tenido una cuota del 34 por 100), pero también Suiza, Holanda y Dinamarca son países con participaciones relevantes en el cable y líderes en penetración de banda ancha.

En el caso de España, aunque la participación del cable no es reducida, ésta pierde peso en los últimos años. Hay que considerar que han tenido dificultades financieras al no haber compartido, al principio de la liberalización, la obra civil existente del antiguo monopolio, algo que ya estaba previsto en la normativa comunitaria y nacional ${ }^{14}$. Por el contrario, al no contar con el «músculo financiero» de Telefónica, utilizan en algunas zonas la propia infraestructura del operador histórico.

\subsection{Una propuesta para medir la competencia entre tecnologías}

La situación de competencia en el mercado de banda ancha de los distintos países se puede hacer de dos formas: mediante el peso del cable en el total de la banda ancha $\left(\mathrm{w}_{2}\right)$ o mediante un índice de concentración, donde se ponderaría no sólo la participación del cable $\mathrm{w}_{2}$, sino también la de las otras tecnologías $\left(\mathrm{w}_{1}=\mathrm{xDSL} \mathrm{y}\right.$ $\mathrm{w}_{3}=$ fibra/LAN). Esto es conveniente distinguirlo en países donde el peso del cable es cercano al óptimo, del 33 por 100 (cuotas de mercado igualitarias de xDSL, cable y fibra), y en otros donde aún persiste desigualdad en las tecnologías restantes. El índice $\mathrm{R}_{\mathrm{Me}}$ de concentración estandarizado estará entre 0 y 1: el 0 refleja un peso equilibrado en las participaciones de las tecnologías en el total de la banda ancha (plena competencia de tecnologías), mientras que el 1 refleja la concentración y dominio total de una de ellas (monopolio de una tecnología), generalmente, la de xDSL del operador histórico. Los índices $\mathrm{R}_{\mathrm{M}}$ sin estandarizar y estandarizado son ${ }^{15}$ :

$$
R_{M}=\sum_{i=1}^{n} w_{i} \cdot\left[\frac{w_{i}}{l_{i}}\right] \quad R_{M e}=\frac{R_{M}-1}{n-1}
$$

\footnotetext{
${ }^{13}$ Exactamente el modelo $\mathrm{BA}_{\mathrm{i}}=\beta_{1}+\beta_{2} \mathrm{w}_{2 \mathrm{i}}+\beta_{3} \mathrm{w}_{2 \mathrm{i}}^{2}+\mathrm{u}_{\mathrm{i}}$ estimado es: $\mathrm{BA}_{\mathrm{i}}=13,3+53,8 \mathrm{w}_{2 \mathrm{i}}-72,7 \mathrm{w}_{2 \mathrm{i}}{ }^{2}$ al derivar éste para obtener su máximo se tiene que: $\frac{d(B A)}{d w_{2}}=0 \Rightarrow w_{2}^{o}=\frac{\hat{\beta}_{2}}{2 \hat{\beta}_{3}} \Rightarrow 36 \%$, un estimador cercano al 33 por 100 esperado. Por supuesto, la variable proxy de la competencia se integrará con otras variables de control (PIBpc, densidad poblacional, etc.) en el análisis econométrico ulterior para verificar su validez.

14 Así, la Directiva 97/33/CE del Parlamento Europeo y del Consejo de 30 de junio ya contemplaba de modo general el uso compartido de instalaciones, y el R.D. 1736/1998 de 31 de julio se refería claramente al uso compartido de las infraestructuras (Art. 48.1).

15 El índice $\mathrm{R}_{\mathrm{M}}$, en su estructura, utiliza las llamadas razones de ventaja (wi/li). Si la razón de ventaja es igual a la unidad, la tecnología participa de forma igualitaria, pero si es mayor (o menor), esa unidad estaría monopolizando (o cediendo mercado). El índice es entonces una sumatoria ponderada de razones de ventaja.
} 
Las participaciones de la tecnología «i» en el total de banda ancha definen las $\left(\mathrm{w}_{\mathrm{i}}\right)$; éstas se contrastan respecto al nivel de perfecta competencia $\left(\mathrm{l}_{\mathrm{i}}\right)$. Si existen «n» tecnologías, y ante plena competencia, éstas deberían tener un $l_{i}=1 / n$. El valor inferior y superior de este índice son $1 \mathrm{y}$ n respectivamente, lo que redefine su estandarización (Cortés y Rubalcava, 1984, 77). Con ello se cumple que $0 \leq \mathrm{R}_{\mathrm{Me}} \leq 1$. Este indicador tiene ventajas estadísticas sobre el de Herfindahl-Hirschman, ya que cumple la propiedad Dalton-Pigou, cambio relativo y es altamente sensible. La primera propiedad garantiza que el índice se eleva ante un incremento real de la desigualdad; en este caso, si se eleva la monopolización de una tecnología. La segunda garantiza que, ante un cambio en la desigualdad, el índice responderá de forma diferenciada, dependiendo de si la participación cedida proviene de una tecnología en monopolio o de una tecnología alternativa. Por último, la sensibilidad se refiere a que el índice reacciona de forma no lineal, incrementándose más en los extremos cuando la distribución se acerca a la competencia igualitaria o al monopolio.

\section{Explicación del grado de penetración de Banda Ancha en la OCDE}

\subsection{Análisis econométrico. La relevancia de la competencia}

Justificada la utilización de un índice de competencia, como el sugerido en la anterior sección, se procede a regresar diferentes modelos, añadiendo variables que se han utilizado en otros estudios y que a la vez sirven de control a la variable competencia. Los cinco modelos log-log muestran los estimadores (elasticidades) estadísticamente significativos, añadiendo en todos ellos el estadístico $t$. Se muestra también la inferencia estadística básica correspondiente.

La variable explicada es la penetración de banda ancha por cada 100 habitantes, mientras que las variables explicativas son: Proxy de competencia RMe, la media de los precios (Mbit/s), PIB per cápita, estas dos últimas variables a paridad de poder adquisitivo (PPA), densidad de población y el porcentaje de penetración de BA en los negocios de diez o más empleados (BAN). 
CUADRO 2

REGRESIONES QUE EXPLICAN LA PENETRACIÓN DE BANDA ANCHA (OCDE, 2009)

\begin{tabular}{|c|c|c|c|c|c|c|c|c|c|c|}
\hline $\begin{array}{l}\text { Variable explicada } \\
\text { In (Banda Ancha) }\end{array}$ & Mod.1 & t calc. & Mod.2 & t calc. & Mod.3 & t calc. & Mod.4 & t calc. & $\begin{array}{c}\text { Mod.5 } \\
\text { (robusto) }\end{array}$ & t calc. \\
\hline $\begin{array}{l}\text { Variables explicativas } \\
\text { Constante } \\
\text { ln (RMe) } \\
\text { ln (Media Precio) } \\
\text { ln (PIB per cápita) } \\
\text { ln (Densidad) } \\
\text { ln (BA en Negocios) } \\
\text { Dicotómica } 1 \\
\text { Dicotómica } 2 \\
\mathrm{R}^{2} \\
\mathrm{R}^{2} \text { ajustada } \\
\mathrm{F} \text { (k-1, n-k) } \\
\mathrm{F} \text { calculada } \\
\text { Heterocedasticidad } \\
\text { White no cruz. } \\
\text { Breusch-Pagan } \\
\text { Koenker } \\
\text { Normalidad } \\
\text { Ji-Cuadrada }\end{array}$ & $\begin{array}{c}\mathbf{- 2 , 0 6 6} \\
\mathbf{- 0 , 1 1 9} \\
-0,132 \\
\mathbf{0 , 5 3 3} \\
- \\
- \\
- \\
- \\
\\
0,7321 \\
0,7012 \\
\mathrm{~F}(3,26) \\
23,686\end{array}$ & $\begin{array}{c}-\mathbf{- 1 , 8 7 0} \\
\mathbf{- 2 , 0 7 0} \\
-0,960 \\
\mathbf{7 , 1 8 0} \\
- \\
- \\
- \\
-\end{array}$ & $\begin{array}{c}1,322 \\
\mathbf{- 0 , 0 9 9} \\
\mathbf{- 0 , 2 0 3} \\
\mathbf{0 , 2 4 7} \\
- \\
- \\
\mathbf{- 0 , 4 9 1} \\
- \\
\\
0,8309 \\
0,8038 \\
F(4,25) \\
30,709 \\
\\
0,8543 \\
0,6711 \\
0,6188 \\
0,5051\end{array}$ & $\begin{array}{c}1,050 \\
-\mathbf{2 , 1 3 0} \\
-\mathbf{1 , 7 9 0} \\
\mathbf{2 , 5 8 0} \\
- \\
- \\
-\mathbf{3 , 8 2 0} \\
-\end{array}$ & $\begin{array}{c}1,283 \\
\mathbf{- 0 , 0 8 5} \\
\mathbf{- 0 , 2 4 4} \\
\mathbf{0 , 2 6 9} \\
- \\
- \\
\mathbf{- 0 , 4 8 0} \\
\mathbf{- 0 , 4 9 1} \\
\\
0,8756 \\
0,8497 \\
\mathbf{F}(5,24) \\
33,783 \\
\\
\\
0,3115 \\
0,5704 \\
0,1846 \\
0,9737\end{array}$ & $\begin{array}{c}1,160 \\
-2,070 \\
-2,430 \\
3,190 \\
- \\
- \\
-4,270 \\
-2,940\end{array}$ & $\begin{array}{r}-0,606 \\
\mathbf{- 0 , 0 9 3} \\
\mathbf{- 0 , 2 6 6} \\
\mathbf{0 , 2 2 5} \\
-0,002 \\
\mathbf{0 , 5 4 1} \\
\mathbf{- 0 , 3 9 1} \\
\mathbf{- 0 , 4 1 8} \\
\\
0,8991 \\
0,8670 \\
F(7,22) \\
28,0120 \\
\\
0,6800 \\
0,9254 \\
0,6565 \\
0,5067\end{array}$ & $\begin{array}{r}-0,430 \\
-\mathbf{2 , 3 5 0} \\
-\mathbf{2 , 6 5 0} \\
\mathbf{2 , 7 3 0} \\
-0,080 \\
\mathbf{2 , 2 4 0} \\
-\mathbf{3 , 4 5 0} \\
-\mathbf{2 , 6 0 0}\end{array}$ & $\begin{array}{r}-0,606 \\
\mathbf{- 0 , 0 9 3} \\
\mathbf{- 0 , 2 6 6} \\
\mathbf{0 , 2 2 5} \\
-0,002 \\
\mathbf{0 , 5 4 1} \\
\mathbf{- 0 , 3 9 1} \\
\mathbf{- 0 , 4 1 8} \\
0,8991 \\
0,8670 \\
\mathbf{F}(7,22) \\
57,107\end{array}$ & $\begin{array}{r}-0,520 \\
-\mathbf{2 , 1 3 0} \\
\mathbf{- 3 , 1 9 0} \\
\mathbf{3 , 0 0 0} \\
-0,090 \\
\mathbf{2 , 6 0 0} \\
\mathbf{- 3 , 3 4 0} \\
-\mathbf{7 , 1 3 0}\end{array}$ \\
\hline Fac. de Inf. Varianza & \multicolumn{2}{|c|}{$\begin{array}{l}\text { No hay multi- } \\
\text { colinealidad }\end{array}$} & \multicolumn{2}{|c|}{$\begin{array}{l}\text { No hay multi- } \\
\text { colinealidad }\end{array}$} & \multicolumn{2}{|c|}{$\begin{array}{l}\text { No hay multi- } \\
\text { colinealidad }\end{array}$} & \multicolumn{2}{|c|}{$\begin{array}{l}\text { No hay multi- } \\
\text { colinealidad }\end{array}$} & \multicolumn{2}{|c|}{$\begin{array}{l}\text { No hay multi- } \\
\text { colinealidad }\end{array}$} \\
\hline
\end{tabular}

NOTA: Los coeficientes destacados en negrita indican significatividad, al menos, del 10 por 100. El factor de inflación de varianza es una medida de la multicolinealidad. Un factor por encima de 10 es un síntoma de fuerte asociación entre variables explicativas, que aumentaría el grado de ajuste pero disminuye la significatividad de las explicativas (t bajas). No se encontró en ningún modelo un factor por encima de este valor.

Hay que enfatizar que el índice de competencia, $\mathrm{R}_{\mathrm{Me}}$, es estadísticamente significativo en todos los modelos y que su elasticidad es relativamente estable. En el Modelo 1 incluso el precio parece no tener impacto estadístico en el crecimiento de la banda ancha; aun así, el modelo explica el 73 por 100 de los cambios en la variable explicada y, en conjunto, el modelo es significativo a partir de la prueba $\mathrm{F}$.

El Modelo 2 utiliza una variable dicotómica que divide en dos grupos a los países de la OCDE, de modo que asume el valor de uno cuando se trata de países con menos desarrollo y de cero en otro caso. Esta distinción se justifica no sólo por la agrupación encontrada en las FDP del Gráfico 3 sino también porque entre países existen bien diferenciadas las funciones de producción, los perfiles de demanda y el consumo de tecnologías. De este modo se obtiene una mejor bondad de ajuste, $\mathrm{R}^{2}$ corregida de 80,3 por 100 . Ahora los tres factores: competencia, precio y poder de compra son significativos, resultando que, en efecto, el nivel diferencial de BA de los países subdesarrollos es más bajo y significativo $(-0,40)$. 
El Modelo 3 añade una variable ficticia que elimina outliers (Irlanda, con un bajo perfil competitivo entre tecnologías), la cual resulta significativa y arroja un mejor ajuste corregido, de prácticamente el 85 por 100. Los Modelos 4 y 5 añaden dos variables que se considera que afectan también al nivel de penetración de banda ancha: la densidad de población y la utilización de esta tecnología en los negocios (BAN).

El Modelo 5 presenta desviaciones típicas robustas que hace eficientes a las elasticidades estimadas. De forma ilustrativa, la lectura de éstas es la siguiente: si se incrementara en un 10 por 100 el nivel del índice $\mathrm{R}_{\mathrm{Me}} \mathrm{y}$ de los precios, la BA decrecería en prácticamente un 1 por 100 y un 2.6 por 100 respectivamente; del mismo modo, si se incrementara el PIB per cápita en un 10 por 100 , la BA aumentará en un 2.2 por 100 y un 5.4 por 100 si lo hiciera en ese porcentaje la variable BAN. La densidad de población no es una variable significativa aunque si lo es la BAN. Los modelos no presentan heterocedasticidad ni multicolinealidad (probada a través del factor de inflación de varianza) y parecen mostrar normalidad en los residuos de acuerdo a los valores de probabilidad de cada una de las pruebas que se muestran arriba.

Concluyendo, econométricamente se observa que una variable de competencia como la sugerida en este trabajo es importante en la explicación de la penetración de la Banda Ancha en un país. La relativa flexibilidad de implementación, de esta variable sobre las otras (políticas de precios, desarrollo económico y empresarial, densidad poblacional), le impone una importancia aún mayor.

\subsection{El método DEA. La eficiencia de los recursos para incrementar la BA}

Se ha analizado hasta ahora el conjunto de los países de la OCDE y se ha mostrado que la competencia entre distintas plataformas es un factor relevante. Ahora corresponde realizar un análisis de la eficiencia en la penetración en BA, tomando la información en conjunto pero arrojando resultados para cada país. El análisis por MCO toma como referencia a la medida promedio, pero, como se ha visto, existen grupos en el interior de la OCDE.

El análisis DEA no toma como referencia al promedio sino a las unidades o países más eficientes en cada uno de los factores; de este modo, cada país más eficiente en cada uno de los factores que afectan a la BA es un punto de referencia, y la combinación de todas estas referencias forman la llamada frontera de eficiencia. En la medida en que un país tiene una distancia sobre esta frontera tiene una ineficiencia. El método DEA mide estas distancias. La noción detallada de la eficiencia no paramétrica y del método DEA se desarrollan al final del artículo en un anexo.

No obstante, la comprensión del concepto de eficiencia bajo dos insumos (precio y PIB per cápita) y un producto ( por $100 \mathrm{BA}$ ) es muy intuitiva. La eficiencia obtenida con el método DEA se infiere de inmediato, dada una frontera determinada por Corea y Suiza, que son los que requieren menos de los dos insumos para obtener una unidad de Banda Ancha. Poniendo el ejemplo de España y con datos reales de la OCDE, la distancia 0B determina la cantidad de los dos insumos para obtener una uni- 
dad de por 100 de BA, una magnitud muy por encima de los referentes Corea y Suiza. La distancia $\mathrm{AB}$ es la ineficiencia de España; queda claro que si $\mathrm{AB}$ tiende a cero (un acercamiento a la frontera) la eficiencia técnica medida como $0 \mathrm{~A} / 0 \mathrm{~B}$ tendería a 1.

\section{GRÁFICO 5}

LA EFICIENCIA TÉCNICA EN EL CASO DE DOS INSUMOS Y UN PRODUCTO. PRECIOS MEDIOS, PIB PER CÁPITA Y BANDA ANCHA EN LA OCDE

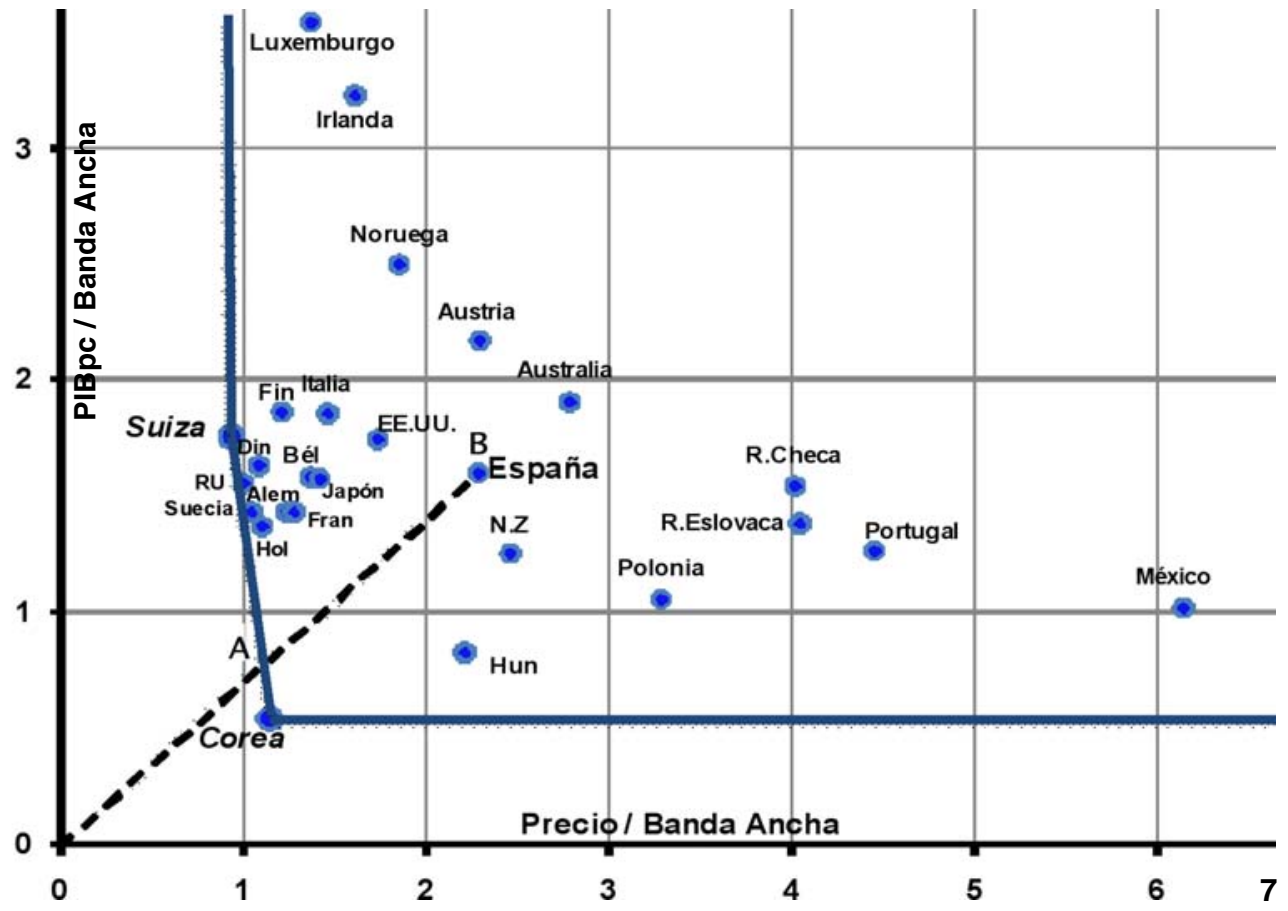

FUENTE: Elaboración propia con datos de la OCDE.

Encontrando las ecuaciones de la recta entre Suiza y Corea y entre el origen y B, se puede indagar el punto $\mathrm{A}$. Con tres puntos: el origen, $\mathrm{A}$ y B, luego tres distancias: OA, OB y AB, éstas son: 1,369, 2,788 y 1,419 respectivamente. Entonces, la eficiencia técnica para España, utilizando como referencia la combinación lineal de insumos óptimos de Corea y Suiza es $0 \mathrm{~A} / 0 \mathrm{~B}=0,491$. De este modo, la magnitud en la que debe reducir sus insumos para situarse en la frontera es de $\mathrm{AB} / 0 \mathrm{~B}=0,509$. Las distancias anteriores se infieren con el simple teorema de Pitágoras y en un plano cartesiano común. Sin embargo, para más variables, bajo $\mathbf{x}$ insumos y $\mathbf{m}$ productos, esto implica resolver un problema de programación lineal. La metodología DEA permite precisamente el cómputo de estas distancias para cada país en un hiperplano ${ }^{16}$.

16 No obstante la utilidad de esta metodología, deben tenerse en cuenta sus límites. Siguiendo a GUARNIDO et al., (2007), no existen criterios para la selección de las variables. La metodología no es muy discriminante, posibilitando que varias unidades sean eficientes cuando puede que no lo sean. Es sensible a outliers sobre todo en las unidades de referencia. Por ello, los resultados del DEA han de 
El anterior gráfico muestra además la gran proximidad a la frontera de Dinamarca, Holanda y Suecia. Sin embargo, esto se desarrolla sólo bajo dos insumos. Del análisis econométrico, se retomarán algunas de las variables relevantes en la explicación de la BA. Como el interés es obtener la máxima BA dados unos insumos, el análisis se desarrollará no con minimización de insumos sino bajo maximización del producto.

El análisis DEA se realiza nuevamente considerando, de igual modo que en el modelo econométrico, como producto a la Banda Ancha y por insumos a la medida de competencia $\mathrm{R}_{\mathrm{Me}}$, al precio medio, al PIB per cápita, a PPA y al por 100 de BA en los negocios. A continuación se muestran los resultados obtenidos (Gráfico 6 y Cuadro 3).

\section{GRÁFICO 6}

\section{EFICIENCIA (PORCENTAJE) Y PENETRACIÓN DE BANDA ANCHA} (PORCENTAJE, OCDE, 2009)

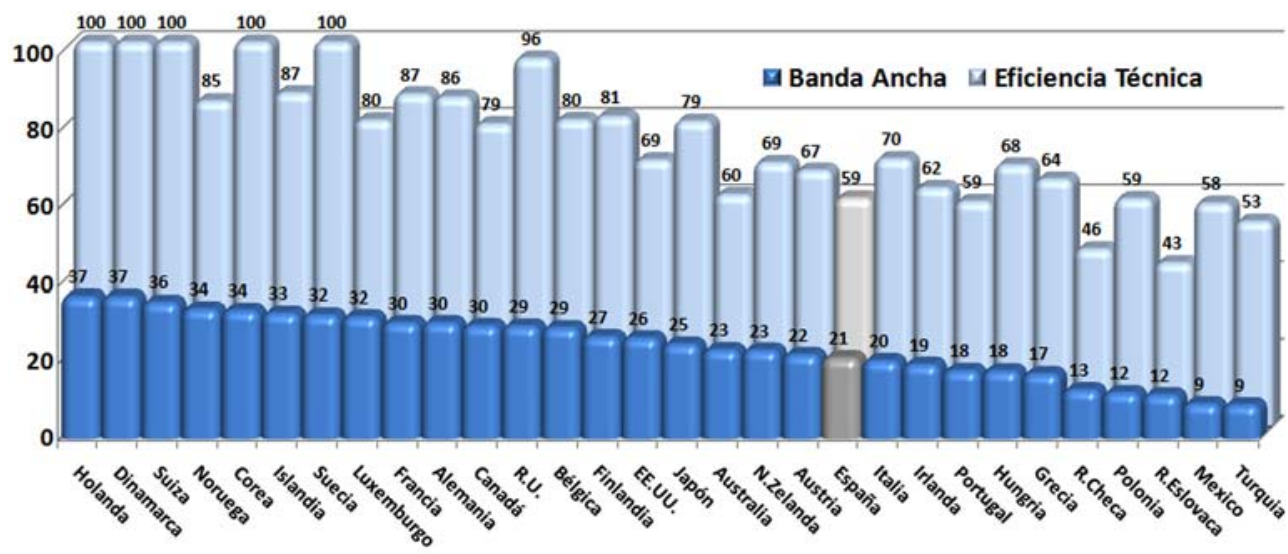

FUENTE: Elaboración propia con datos de la OCDE. Por datos parciales se omite a Chile.

El análisis de eficiencia técnica radial (Gráfico 6) nos indica que existe una correlación de Pearson alta entre este índice y la penetración de banda ancha $(\mathrm{R}=91,5$ por 100). Países como Holanda, Dinamarca, Suiza, Corea y Suecia son eficientes y se sitúan en la frontera. Es notable, además, que estos países rondaban alrededor de una participación del 33 por 100 óptimo en cable $\left(\mathrm{w}_{2}\right)$, de acuerdo a lo presentado en el Gráfico 4 (Corea y Suiza son lo más cercanos a este óptimo). El Reino Unido, Islandia, Francia, Alemania se posicionan muy cerca; más alejados están países como Noruega, Canadá, EE.UU., y España; en un cuarto grupo se sitúan países predominantemente del este europeo, México y Turquía.

tomarse con cierta cautela. Desarrollos actuales como el VEA(Value Efficiency Analysis) y la programación multicriterio intentan solucionar algunas de éstas dificultades. Una referencia reciente de aplicación del DEA a las telecomunicaciones es GIOKAS (2008), quien emplea DEA y el Proceso Analítico Jerárquico a países de la OCDE, bajo insumos y productos diferentes a los del presente trabajo, y que dificultan cualquier comparación. Por su parte, GRUBESIC (2010) y HAYNES et al., (2010) realizan un estudio DEA para EE.UU. con conjuntos diferentes de insumos y productos sin incluir a la competencia. 
CUADRO 3

VALORES ACTUALES Y EFICIENTES DE PRODUCTO E INSUMOS OCDE, 2009

\begin{tabular}{|c|c|c|c|c|c|c|c|c|c|c|c|c|}
\hline \multirow{2}{*}{$\begin{array}{c}\text { Rank } \\
\text { en } \\
\text { BA }\end{array}$} & \multirow{2}{*}{ País } & \multirow{2}{*}{$\begin{array}{c}\text { Eficiencia } \\
\text { DEA }\end{array}$} & \multicolumn{6}{|c|}{ Valor original } & \multicolumn{4}{|c|}{ Valor proyectado } \\
\hline & & & BA & $\mathbf{R}_{\mathrm{Me}}$ & Precios & PIBpc & BAN & BA & $\mathbf{R}_{\mathrm{Me}}$ & Precios & PIBpc & BAN \\
\hline 1 & Holanda & 100 & 37,1 & 25,1 & 41,0 & 50.868 & 87,4 & 37,1 & 25,1 & 41,0 & 50.868 & 87,4 \\
\hline 2 & Dinamarca & 100 & 37,1 & 18,3 & 40,2 & 60.390 & 82,3 & 37,1 & 18,3 & 40,2 & 60.390 & 82,3 \\
\hline 3 & Suiza & 100 & 35,6 & 36,0 & 33,3 & 62.577 & 100,0 & 35,6 & 36,0 & 33,3 & 62.577 & 100,0 \\
\hline 4 & Noruega & 85 & 33,9 & 19,3 & 62,8 & 84.678 & 90,0 & 40,0 & 19,3 & 43,5 & 63.553 & 90,0 \\
\hline 5 & Corea & 100 & 33,5 & 6,4 & 38,5 & 18.202 & 97,2 & 33,5 & 6,4 & 38,5 & 18.202 & 97,2 \\
\hline 6 & Islandia & 87 & 32,8 & 81,5 & 40,6 & 46.334 & 99,5 & 37,8 & 23,4 & 40,6 & 46.334 & 99,5 \\
\hline 7 & Suecia & 100 & 32,4 & 11,0 & 32,5 & 50.431 & 89,3 & 32,4 & 11,0 & 32,5 & 50.431 & 89,3 \\
\hline 8 & Luxemburgo & 80 & 31,9 & 57,8 & 43,5 & 112.765 & 88,8 & 40,0 & 19,7 & 43,4 & 65.159 & 88,8 \\
\hline 9 & Francia & 87 & 30,4 & 84,2 & 37,5 & 43.453 & 92,9 & 35,1 & 22,1 & 37,5 & 43.453 & 92,9 \\
\hline 10 & Alemania & 86 & 30,3 & 73,2 & 38,9 & 43.484 & 89,2 & 35,4 & 21,4 & 38,9 & 43.484 & 89,2 \\
\hline 11 & Canadá & 79 & 29,6 & 25,9 & 54,2 & 42.945 & 94,3 & 37,6 & 20,4 & 42,0 & 42.945 & 94,3 \\
\hline 12 & R.U. & 96 & 29,5 & 50,0 & 30,8 & 42.275 & 88,3 & 30,8 & 23,1 & 30,8 & 42.275 & 87,3 \\
\hline 13 & Bélgica & 80 & 29,0 & 25,2 & 39,8 & 46.002 & 91,4 & 36,4 & 22,8 & 39,8 & 46.002 & 91,4 \\
\hline 14 & Finlandia & 81 & 26,7 & 57,2 & 32,5 & 49.728 & 93,9 & 33,2 & 27,8 & 32,5 & 49.728 & 93,7 \\
\hline 15 & EE.UU. & 69 & 26,4 & 18,2 & 45,8 & 46.008 & 94,3 & 38,1 & 18,2 & 42,4 & 46.008 & 94,3 \\
\hline 16 & Japón & 79 & 24,8 & 12,5 & 35,2 & 39.081 & 76,6 & 31,2 & 12,5 & 34,5 & 39.081 & 76,6 \\
\hline 17 & Australia & 60 & 23,3 & 55,1 & 64,8 & 44.223 & 96,6 & 38,6 & 21,0 & 43,1 & 44.223 & 96,6 \\
\hline 18 & N. Zelanda & 69 & 23,2 & 81,6 & 57,1 & 29.031 & 91,0 & 33,8 & 12,7 & 38,3 & 29.031 & 91,0 \\
\hline 19 & Austria & 67 & 22,1 & 34,6 & 50,6 & 47.767 & 76,8 & 33,1 & 20,7 & 36,4 & 47.767 & 76,8 \\
\hline 20 & España & 59 & 21,2 & 52,9 & 48,3 & 33.955 & 93,8 & 35,7 & 15,4 & 40,2 & 33.955 & 93,8 \\
\hline 21 & Italia & 70 & 20,5 & 92,1 & 29,9 & 37.936 & 83,8 & 29,4 & 20,4 & 29,9 & 37.936 & 83,7 \\
\hline 22 & Irlanda & 62 & 19,5 & 55,4 & 31,3 & 62.806 & 80,2 & 31,4 & 24,8 & 31,3 & 53.414 & 80,2 \\
\hline 23 & Portugal & 59 & 17,9 & 25,0 & 79,5 & 22.574 & 84,7 & 30,6 & 9,3 & 34,8 & 22.574 & 84,7 \\
\hline 24 & Hungría & 68 & 17,8 & 15,8 & 39,4 & 14.707 & 75,6 & 26,2 & 5,3 & 30,0 & 14.707 & 75,6 \\
\hline 25 & Grecia & 64 & 17,0 & 99,7 & 27,7 & 29.634 & 84,2 & 26,5 & 15,4 & 27,7 & 29.634 & 75,6 \\
\hline 26 & R. Checa & 46 & 12,9 & 16,9 & 51,6 & 19.858 & 77,7 & 27,9 & 8,1 & 31,8 & 19.858 & 77,7 \\
\hline 27 & Polonia & 59 & 12,1 & 33,4 & 39,8 & 12.810 & 58,3 & 20,5 & 4,9 & 23,4 & 12.810 & 58,3 \\
\hline 28 & R. Eslovaca & 43 & 11,6 & 16,2 & 46,8 & 16.023 & 78,2 & 27,3 & 5,9 & 31,2 & 16.023 & 78,2 \\
\hline 29 & México & 58 & 9,2 & 38,5 & 56,7 & 9.422 & 45,6 & 15,9 & 3,5 & 18,2 & 9.422 & 45,6 \\
\hline 30 & Turquía & 53 & 9,0 & 89,6 & 135,4 & 9.125 & 81,8 & 16,8 & 3,2 & 19,3 & 9.125 & 48,7 \\
\hline
\end{tabular}

FUENTE: OCDE, 2009.

¿Pero en cuánto estarían determinando la eficiencia radial sus inputs? El Cuadro 3 muestra los valores originales y proyectados del producto (banda ancha) y los insumos ( $\mathrm{R}_{\mathrm{Me}}$, precios, PIBpc y la banda ancha en los negocios). Los valores proyectados o eficientes se han obtenido de la metodología DEA orientada a la maximización 
CUADRO 4

CAMBIOS DE LOS COMPONENTES HACIA LA EFICIENCIA (PORCENTAJE, OCDE, 2009)

\begin{tabular}{|c|c|c|c|c|c|c|c|}
\hline \multirow{2}{*}{$\begin{array}{r}\text { Rank } \\
\text { en BA }\end{array}$} & \multirow[b]{2}{*}{ País } & \multirow{2}{*}{$\begin{array}{c}\text { Eficiencia } \\
\text { DEA }\end{array}$} & \multicolumn{5}{|c|}{ Cambio $\%$} \\
\hline & & & $\begin{array}{l}\text { Banda } \\
\text { Ancha }\end{array}$ & RMe & Precio & PIBpc & $\begin{array}{c}\text { BA } \\
\text { negocios }\end{array}$ \\
\hline 1 & Holanda & 100 & 0,0 & 0,0 & 0,0 & 0,0 & 0,0 \\
\hline 2 & Dinamarca & 100 & 0,0 & 0,0 & 0,0 & 0,0 & 0,0 \\
\hline 3 & Suiza & 100 & 0,0 & 0,0 & 0,0 & 0,0 & 0,0 \\
\hline 4 & Noruega & 85 & 18,1 & 0,0 & $-30,6$ & $-24,9$ & 0,0 \\
\hline 5 & Corea & 100 & 0,0 & 0,0 & 0,0 & 0,0 & 0,0 \\
\hline 6 & Islandia & 87 & 15,3 & $-71,3$ & 0,0 & 0,0 & 0,0 \\
\hline 7 & Suecia & 100 & 0,0 & 0,0 & 0,0 & 0,0 & 0,0 \\
\hline 8 & Luxemburgo & 80 & 25,5 & $-65,9$ & $-0,4$ & $-42,2$ & 0,0 \\
\hline 9 & Francia & 87 & 15,7 & $-73,8$ & 0,0 & 0,0 & 0,0 \\
\hline 10 & Alemania & 86 & 16,7 & $-70,8$ & 0,0 & 0,0 & 0,0 \\
\hline 11 & Canadá & 79 & 27,1 & $-21,2$ & $-22,5$ & 0,0 & 0,0 \\
\hline 12 & Reino Unido & 96 & 4,4 & $-53,8$ & 0,0 & 0,0 & $-1,2$ \\
\hline 13 & Bélgica & 80 & 25,3 & $-9,5$ & 0,0 & 0,0 & 0,0 \\
\hline 14 & Finlandia & 81 & 24,1 & $-51,4$ & 0,0 & 0,0 & $-0,2$ \\
\hline 15 & EE.UU. & 69 & 44,3 & 0,0 & $-7,4$ & 0,0 & 0,0 \\
\hline 16 & Japón & 79 & 25,9 & 0,0 & $-1,8$ & 0,0 & 0,0 \\
\hline 17 & Australia & 60 & 65,7 & $-61,9$ & $-33,5$ & 0,0 & 0,0 \\
\hline 18 & Nueva Zelanda & 69 & 45,8 & $-84,4$ & $-32,9$ & 0,0 & 0,0 \\
\hline 19 & Austria & 67 & 50,2 & $-40,2$ & $-27,9$ & 0,0 & 0,0 \\
\hline 20 & España & 59 & 68,3 & $-70,9$ & $-16,9$ & $\mathbf{0 , 0}$ & $\mathbf{0 , 0}$ \\
\hline 21 & Italia & 70 & 43,8 & $-77,9$ & 0,0 & 0,0 & $-0,2$ \\
\hline 22 & Irlanda & 62 & 61,1 & $-55,2$ & 0,0 & $-15,0$ & 0,0 \\
\hline 23 & Portugal & 59 & 70,9 & $-62,8$ & $-56,3$ & 0,0 & 0,0 \\
\hline 24 & Hungría & 68 & 47,0 & $-66,5$ & $-23,7$ & 0,0 & 0,0 \\
\hline 25 & Grecia & 64 & 55,5 & $-84,6$ & 0,0 & 0,0 & $-10,2$ \\
\hline 26 & República Checa & 46 & 116,7 & $-52,1$ & $-38,5$ & 0,0 & 0,0 \\
\hline 27 & Polonia & 59 & 69,1 & $-85,3$ & $-41,2$ & 0,0 & 0,0 \\
\hline 28 & Rep. Eslovaca & 43 & 135,2 & $-63,6$ & $-33,3$ & 0,0 & 0,0 \\
\hline 29 & México & 58 & 72,3 & $-90,9$ & $-67,9$ & 0,0 & 0,0 \\
\hline 30 & Turquía & 53 & 87,5 & $-96,4$ & $-85,7$ & 0,0 & $-40,4$ \\
\hline \multicolumn{2}{|c|}{ Media pond. (con población) } & $74,85 \%$ & $37,88 \%$ & $-53,10 \%$ & $-17,99 \%$ & $-3,34 \%$ & $-2,61 \%$ \\
\hline
\end{tabular}


del producto, de modo que el producto banda ancha puede verse como el resultado al que se llegaría potenciando los insumos como lo efectúan los países más eficientes.

Es de interés determinar el porcentaje de cambio de los productos e insumos respecto a la frontera eficiente; de este modo, la metodología utilizada podría darnos indicios sobre qué componentes pesan más en cada país en particular. Véase Cuadro 4.

En el caso de España, ha resultado evidente que el componente más importante es la competencia, siguiéndole los precios ${ }^{17}$. Esta sugerencia se extiende, de hecho, al grupo de países de la $\mathrm{OCDE}^{18}$, ya que la variable $\mathrm{R}_{\mathrm{Me}}$ debe caer en un 53 por 100 y los precios en un 17 por 100 , es decir, debe incrementarse la competencia entre plataforma tecnológicas para trasladarse a índices $\mathrm{R}_{\mathrm{Me}}$ más bajos. Para España, la penetración actual de banda ancha de 21,2 por 100 podría elevarse al 35,7 por 100 (muy cercano a los niveles de los países punteros) de utilizarse eficientemente los insumos. De forma específica, la competencia debería intensificarse, modificando su índice $\mathrm{R}_{\mathrm{Me}}$ actual de 52,9 por 100 a un 15,4 por 100 y bajando los precios de la media actual de 48,3 dólares a PPA en 40,2 dólares. Esto implica reducir en casi un 71 por 100 al primer insumo y un 17 por 100 al segundo; el PIB per cápita, en el contexto de la eficiencia no paramétrica, no necesita modificarse.

El caso de Noruega es significativo porque, a pesar de situarse en la cuarta posición del ranking de penetración de banda ancha, no obtiene la máxima eficiencia. Esto se debe a que sus insumos están subutilizados respecto a los referentes de los países más eficientes como Corea, Suiza, Dinamarca y Holanda. Los precios de Noruega deberían bajar en un 30,6 por $100^{19}$ (Cuadro 4). Por último, de los cuatro países con más suscriptores de la OCDE: EE.UU, Japón, Alemania y Francia, el análisis sugiere que los dos primeros deberían hacer uso de políticas de precios para reducirlos, mientras que los dos últimos deben centrar sus esfuerzos en intensificar más la competencia ${ }^{20}$.

17 En España hay una reducida competencia, en el sentido de verdaderas alternativas de red a la del ex monopolio, ya que el cable pierde peso en los últimos años. Los precios, tal y como indica la CMT en sus informes (CMT, 2010) son altos, pero este factor no es tan importante como la falta de competencia entre verdaderas alternativas. A este resultado se llega en buena parte de los países de la OCDE, a saber, que la competencia entre tecnologías distintas es más importante que las simples bajadas de precios para el desarrollo de la banda ancha (RUBIO, 2010).

18 Nótese que el tipo de competencia que se defiende en el artículo es el de competencia inter- tecnologías (o inter-plataformas), que es la que estimula, de acuerdo con la literatura económica, la penetración de banda ancha, más que la competencia intra-plataforma (ver, además de los trabajos citados en el apartado 2, los estudios econométricos recientes de BOUKAERT et al., (2010) y RUBIO (2010).

19 Noruega es, tradicionalmente, un país con precios altos en banda ancha (precio por Mbits), aunque también es un país de alto PIB por habitante.

20 Y es que EE.UU. y Japón tienen «suficiente» competencia entre distintas tecnologías (el primer país con los operadores de cable y el segundo con los nuevos operadores de fibra óptica), pero unos precios relativamente altos que frenan la penetración de banda ancha. En cambio, los dos europeos son países donde la competencia está basada en la tecnología de sus operadores históricos (DSL), es decir, además de tener cuotas de mercado importantes, la competencia existente tiene una dependencia crítica de la infraestructura del ex monopolio. Motivo por el cual la creación de verdaderas alternativas de red (competencia entre tecnologías, interplataformas) se traduciría en incrementos de penetración y, en 


\section{Conclusiones y sugerencias}

Una de las cuestiones que más ha llamado la atención en el proceso de liberalización de las telecomunicaciones europeas es que más de diez años después de producirse ésta en servicios e infraestructuras esté aún debatiéndose la liberalización de las mismas. Esto es algo decidido en su momento pero que no se implementó del todo en determinados países, que pusieron énfasis en la apertura del bucle de la infraestructura del operador histórico y en las bajadas de precios. Este último modelo, de competencia restringido a los servicios, no ha propiciado el desarrollo de verdaderas alternativas de red que dinamicen el mercado. Por el contrario, el modelo basado en la competencia desde plataformas tecnológicas distintas, tal y como se ha mostrado en el análisis econométrico y análisis DEA realizados en este trabajo, ha supuesto una mayor expansión de la banda ancha.

El trabajo ha encontrado evidencia de que la competencia entre diferentes plataformas resulta estadísticamente significativa para explicar el aumento de la BA. Se ha encontrado también, en el análisis DEA, que el factor que más debería cambiar en los países de la OCDE es la competencia antes que los precios.

En particular, la medición de la eficiencia a través del análisis DEA proporciona una orientación sobre dónde y en qué medida deben agilizarse acciones de política económica para acercarse a los países líderes en la penetración de Banda Ancha.

El resultado para España es bastante ilustrativo. Con una penetración de BA del 21,2 por 100 en 2009, una política que intensificara la competencia entre las diferentes tecnologías (aún permaneciendo casi constantes el resto de «insumos», es decir, el PIB per cápita y los precios ${ }^{21}$ ) se reflejaría en una considerable mejora, ya que, de seguir las pautas de los países de referencia, su penetración en BA se ubicaría en un 35,7 por 100, bastante próxima al de los países líderes (en 2009 Holanda y Dinamarca tuvieron ambos una penetración de 37,1 por 100).

Para terminar, y si tuviéramos que extractar las medidas necesarias para mejorar la situación de la banda ancha en España, cabría centrarlas en las siguientes:

- Cambiar el enfoque regulatorio hacia un modelo de competencia más sostenible a medio y largo plazo, basado en la existencia de verdaderas alternativas de red que tengan incentivos a invertir e innovar en sus propias redes para la difusión de sus servicios propios de banda ancha.

general, en un mayor desarrollo del mercado de banda ancha. Hay que indicar que el regulador francés (Arcep) está apostando desde 2007, de cara a las Redes de Nueva Generación, por abrir las infraestructuras de conductos a otros operadores dispuestos a invertir en red propia mediante fibra óptica. De igual manera, el regulador alemán viene instando al operador dominante a permitir a los competidores acceder a los conductos para que instalen su fibra.

21 Sin perjuicio de que la banda ancha en España es cara, y especialmente la que ofrece el operador histórico, Telefónica (véase CMT, Comparativa Internacional a junio de 2010, donde se señala que la mejor oferta de Telefónica de banda ancha más voz en el tramo de velocidad media es un 84,6 por 100 superior a la media de las mejores ofertas en Europa. 
- Facilitar la consolidación de los operadores alternativos con la apertura real y efectiva de las infraestructuras civiles, especialmente de cara al futuro desarrollo de las Redes de Acceso de Nueva Generación, de forma que estas sean verdaderas alternativas de red en las plataformas avanzadas de banda ancha.

- Dar más protagonismo al mercado y a sus agentes, y no a la regulación del bucle del operador histórico que, si bien es un medio para obtener competencia, no es el mejor a largo plazo ni puede permanecer indefinidamente.

\section{Referencias bibliográficas}

[1] AGHION, P.; BLOOM, N.; BLUNDELL, R., y GRIFFITH y HOWITT, P. (2005): «Competition and innovation: an inverted-U relationshuip», Quarterly Journal of Economics, vol. 120 (2), pp. 701-728.

[2] BANKER, R.; CHARNES, A. y COOPER, W. (1984): «Some models for estimating technical and scale inefficiencies in Data Envelopment Analysis», Management Science. vol. 30 (9), pp. 1078-1092.

[3] BOUKAERT et al., (2010): «Access regulation, competition and broadband penetration: An international study, Telecommunications Policy, 34, pp. 661-671.

[4] BOURREAU, M.; DOGAN, P. y MANANT, M. (2010): «A critical review of the "ladder of investment" approach», Telecommunications Policy, vol. 34, pp. 683-696.

[5] CAMBINI, C. y JIANG, Y. (2009): «Broadband Investment and Regulation: A literature review», Telecommunications Policy, vol. 33, pp.559-574.

[6] CMT (2010): Informe Anual 2009, julio.

[7] CMT (2010): «Comparativa Internacional en Ofertas Comerciales de Banda Ancha en la Unión Europea y España a junio 2010», diciembre 2010.

[8] CHARNES, A.; COOPER, W. y RHODES E. (1978): «Measurement the efficiency of decision making units», European Journal of Operational Research, vol. 2, pp. 429-444.

[9] CHARNES, A.; COOPER, W.; LEWIN, A. y SEIFORD, L. (1995): Data Envelopment Analysis: Theory, Methodology and Applications, Boston, Kluver Nijhoff Publishing.

[10] CHOONGOK, L. y CHAN-OLMSTEL, S. (2005): «Competitive advantage of broadband internet: A comparative study between South Korea and United States», Telecommunications Policy, vol. 28, pp. 649-677.

[11] COMISIÓN EUROPEA (2004): Comunicado de la Comisión al Consejo, Parlamento Europeo, Comité Económico y Social y Comité de las Regiones-Conectando Europa a alta velocidad: Estrategias Nacionales de Banda Ancha, COM (2004) 369 final.

[12] COMISIÓN EUROPEA (2009): Directrices comunitarias para la aplicación de las normas sobre ayudas estatales al despliegue rápido de redes de banda ancha, DOCE, 2009/C 235/04.

[13] COOPER, W.; SEIFORD, L. y TONE, K. (1999): Data Envelopment Analysis A Comprehensive Text with Models, Applications, References and DEA-Solver Software, Boston, Kluver Nijhoff Publishing.

[14] CORTÉS, F. y RUBALCAVA, R. (1984): Técnica Estadística para el estudio de la desigualdad social, México, El Colegio de México, pp. 72-82. 
[15] DEBREU, G. (1951): «The coeficcient of resource utilisation», Econométrica, 19, pp. 173-292.

[16] DISTASO, W.; LUPI, P. y MANENTI, F. (2006): «Platform competition and broadband uptake: Theory and Empirical evidence from the European Unión», Information Economics and Policy, 18, pp. 87-106.

[17] ESSENACH, J. A. (2008): «Broadband Policy: Does the U.S. Have It Right After All?, Progress \& Freedom Fundation, ed. Electronic, septiembre.

[18] FÄRE, R.; GROSSKOPF, S. y LOVELL, C. (1985): The measurement of efficiency of production, Boston, Kluver Nijhoff Publishing.

[19] FARRELL, M. (1957): «The measurement of productive efficiency», Journal of the Royal Statistical Society (Series A), 120, part. III, pp. 253-290.

[20] FORD, G.; KOUTSKY, T. y SPIWAK, L (2007a): «The Broadband Performance Index: A Policy-Relevant Method of Comparing Broadband Adoption Among Countries», Phoenix Center Policy Paper, n. 29, disponible en: http://www.phoenixcenter.org/pcpp/ PCPP29Final.pdf

[21] FORD, G.; KOUTSKY, T. y SPIWAK, L. (2007b): «The Demographic and Economic Drivers of Broadband Adoption in the United States». Phoenix Center Policy Paper, $\mathrm{n}$. 31 disponible en: http://www.phoenix-center.org/pcpp/PCPP31Final.doc.

[22] FORD, G.; KOUTSKY, T. y SPIWAK, L (2008): «The Broadband Efficiency Index: What Really Drives Broadband Adoption Across the OECD?» Phoenix Center Policy Paper n. 33, disponible en: http://www.phoenix-enter.org/pcpp/PCPP33Final.pdf.

[23] FRIEDERISZICK, H.; MICHAL GRAJEK y LARS-HENDRIK RÖLLER (2008): «Analyzing the Relationship between Regulation and Investment in the Telecom Sector», W.P. 108-01, Berlin, ESMT.

[24] GIOKAS, D. (2008): «Efficiency ranking of the OECD member states in the area of telecommunications: A composite AHP/DEA study», Telecommunications Policy, vol. 32 , pp. 672-685

[25] GIVEN, J. (2010): «Take your partners. Public private interplay in Australian and New Zeland plans for next generation broadband", Telecommunications Policy, vol. 34, pp. 540-549.

[26] GUARNIDO, A. et al., (2007): La desregulación y privatización de las empresas públicas. El caso de las telecomunicaciones en España, Monografías de Ciencias Económicas y Jurídicas de la Universidad de Almería.

[27] GRUBESIC, T. (2010): «Efficiency in broadband service provision: A spatial analysis», Telecommunications Policy, vol. 34, pp. 117-131.

[28] HAYNES, K. y PARAJULI, J. (2010): «Broadband Internet in the United States», Third Annual Conference on Competition and Regulation in Network Industries, disponible en http://www.jcrni.org.

[29] HÖFFLER, F. (2007): «Cost and Benefits from Infrastructure Competition. Estimating Welfare Effects from Broadband Access Competition», Telecommunications Policy, vol. 31, pp. 401-418.

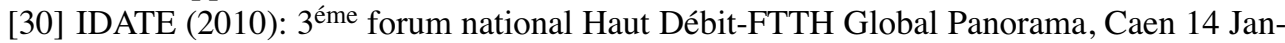
vier 2010, presentación de Roland Montagne, disponible en www.forum-thd.com

[31] KATZ, R. (2008): La competencia entre plataformas: teoría y resultados. Enter, Madrid. 
[32] LECG, Ltd. (2007): «Access Regulation and Infrastructure Investment in the Telecommunications Sector: An Empirical Investigation», estudio para ETNO.

[33] MONTOYA, M. (2007): Independencia y compromiso en la regulación de las telecomunicaciones. Tesis doctoral. UAB. http://www.tdx.cbuc.es/TESIS_UAB/AVAILABLE/TDX-0229108-163938//mamblde1.pdf

[34] RUBIO MARTÍN, J. (2010): Regulación y Defensa de la Competencia: Efectos en el Mercado de las Telecomunicaciones, Tesis Doctoral, Universidad Complutense, Madrid.

[35] SCHEER, F. (1967): «Market Structure and the Employment of Scientists and Engineers», American Economic Review, 57 (3), pp. 524-531.

[36] UIMP (2009): ponencias del XXIII Encuentro de las Telecomunicaciones.

[37] WALLSTEN, S. y HAUSLADEN, S. (2009): «Net neutrality, un bundling and their effects on international investment in next generation networks», Review of Network Economic, 8 (1), pp. 90-112. 


\section{APÉNDICE}

\section{El concepto de Eficiencia}

El concepto de eficiencia viene tratado teóricamente en Debreu (1951) y Koopmans (1951); Farrell (1957) intenta esquematizar estos conceptos, pero no es hasta el trabajo de Charners, Cooper y Rhodes (1978), cuando el método DEA recibe su nombre y empieza a aplicarse en diferentes campos de la economía. Los autores anteriores plantean un modelo orientado a los insumos y bajo rendimiento constante a escala. Posteriormente, otros trabajos modificaron estos supuestos. Banker, Charner y Cooper (1984) generan una propuesta con rendimientos variables a escala.

La eficiencia según el método DEA se descompone en dos elementos: la eficiencia técnica y la eficiencia distributiva; la primera puede verse como el máximo de producto(s) dado(s) un(os) insumo(s), mientras que la segunda se refiere a las proporciones óptimas de los insumos dados los precios de estos; estas dos eficiencias se muestran en el Gráfico A1.

\section{GRÁFICO A1 \\ EL CONCEPTO DE EFICIENCIA}

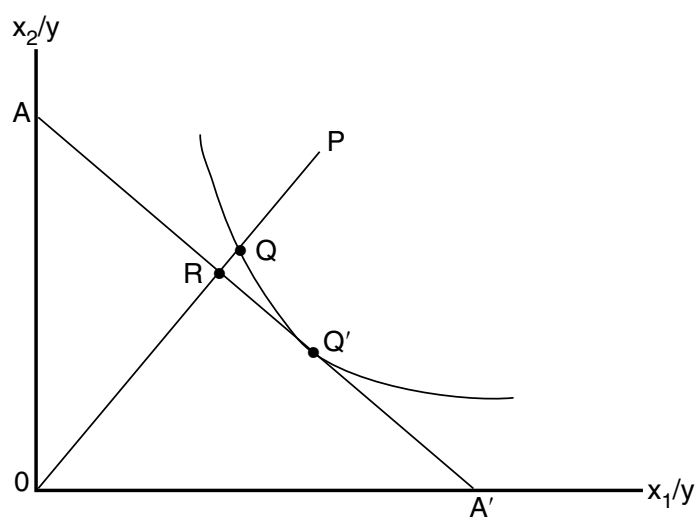

Del esquema anterior se desprende que $\mathrm{P}$ es la cantidad específica de insumos de la empresa para producir una unidad de producto. La distancia QP es la ineficiencia de la empresa. QP/OP es el porcentaje en que debe reducir la empresa sus insumos para llegar a ser eficiente. Ahora bien, ET $=0 \mathrm{Q} / 0 \mathrm{P}$ es la forma de evaluar la eficiencia técnica. Como los segmentos $0 \mathrm{Q}$ y QP son complementarios y forman parte del segmento $0 \mathrm{P}$, en realidad $0 \mathrm{Q} / 0 \mathrm{P}=1-0 \mathrm{Q} / 0 \mathrm{P}$, es decir, la eficiencia es el resultado de restarle a la unidad la ineficiencia. RQ es el segmento que representa lo que deben reducirse los costes para llegar a una proporción óptima de insumos. $\mathrm{Q}$ representa, respecto al punto $\mathrm{P}$, la eficiencia técnica pero con ineficiencia distributiva. Q' representa la eficiencia económica, tanto si es eficiente técnica como dis- 
tributivamente. Por ello, la eficiencia distributiva es: ED = 0R/0Q; es la forma de evaluar la eficiencia distributiva dada una línea de isocoste. La eficiencia económi$c a$ es el producto de las dos eficiencias definidas: $\mathrm{EE}=(\mathrm{ET})(\mathrm{ED})=(0 \mathrm{Q} / 0 \mathrm{P})$ $(0 \mathrm{R} / 0 \mathrm{Q})=0 \mathrm{R} / 0 \mathrm{P}$

Puede encontrarse que las tres medidas de eficiencia se encuentran entre 0 y 1 . La medida de eficiencia puede estar orientada a los insumos o al producto y pueden asumirse rendimientos decrecientes o constantes a escala. Ambas eficiencias son equivalentes sólo cuando existen rendimientos constantes, y son desiguales cuando existen rendimientos decrecientes. Obsérvese que, precisamente, una de las ventajas de este método es que no se precisa que la función de producción tenga un tipo de rendimientos específico.

Hasta ahora se ha supuesto conocida la función de producción de eficiencia y, por tanto, se pueden evaluar otras unidades productivas a través de ella; desde luego, esta función se desconoce y tiene que estimarse. Farrell (1957) proponía dos métodos: el de la isocuanta no paramétrica y el de la función paramétrica; en este trabajo sólo se hace uso del primer método.

\section{El método DEA}

Es el método no paramétrico que realiza una aproximación de la frontera eficiente de Farrell utilizando la programación lineal. El modelo es:

$$
\begin{gathered}
\operatorname{Max}^{\prime} y_{i} / v^{\prime} x_{i} \\
\text { s.a. } u^{\prime} y_{j} / v^{\prime} x_{j} \leq 1, j=1,2, \ldots, n \\
u, v=0
\end{gathered}
$$

Donde: $\mathrm{u}$ es un vector de $\mathrm{m} \times 1$ cuyos elementos son ponderadores de producto, $\mathrm{v}$ es un vector de $\mathrm{K} \times 1$ cuyos elementos son ponderadores de insumos, e y es una matriz de $\mathrm{m} \times \mathrm{n}$ productos. Por otro lado, $\mathrm{x}$ es una matriz de $\mathrm{K} \times \mathrm{n}$ insumos. $\mathrm{K}$ es el número de insumos de la unidad productora, $\mathrm{m}$ es el número de productos de la unidad productora y $\mathrm{n}$ es el número de unidades productoras. Sin embargo, en el problema de programación lineal anterior existe un número infinito de soluciones; esto puede solucionarse incorporando la restricción de que los insumos ponderados sean iguales a $1, v^{\prime} x_{i}=1$.

$$
\begin{gathered}
\operatorname{Max} \mu, \mathrm{v}\left(\mu^{\prime} \mathrm{y}_{\mathrm{i}}\right) \\
\mathrm{s} . \mathrm{a} \cdot \mathrm{v}^{\prime} \mathrm{x}_{\mathrm{i}}=1 \\
\mu^{\prime} \mathrm{y}_{\mathrm{j}}-\mathrm{v}^{\prime} \mathrm{x}_{\mathrm{j}} \leq 0, \mathrm{j}=1,2, \ldots, \mathrm{n} \\
\mu, \mathrm{v}=0
\end{gathered}
$$

Donde $\mu^{\prime} \mathrm{y} \mathrm{v}^{\prime}$, que eran las medidas de eficiencia de la unidad evaluada, se transforman en $\mu$ y v. Este problema de programación lineal (PPL) recibe el nombre de 
solución multiplicativa. El dual de este problema es el siguiente:

$$
\begin{gathered}
\text { Min }_{\theta, \lambda} \theta \\
\text { s.a. }-\mathrm{y}_{\mathrm{i}}+\mathrm{Y} \lambda \geq 0 \\
\theta \mathrm{x}_{\mathrm{i}}-\mathrm{X} \lambda \geq 0 \\
\lambda \geq 0
\end{gathered}
$$

Donde $\theta$ es un escalar y $\lambda$ es un vector de $\mathrm{n} \times 1$ de constantes. Este planteamiento tiene menos restricciones que el anterior PPL, $\mathrm{K}+\mathrm{m}<\mathrm{n}+1$. Como ha de evaluarse la eficiencia para cada unidad, el PPL se ejecuta tantas veces como países.

\section{Funciones de densidad de probabilidad (FDP)}

La función de densidad de probabilidad para los 30 países puede calcularse, suponiendo una distribución normal y ponderándola con la participación de la población de cada país: $F(x)=\sum_{i=1}^{n=30} \grave{u}_{i} \cdot\left[\frac{1}{\sigma \sqrt{2 \pi}} \cdot e^{\frac{-\left(x-\mu_{i}\right)}{2 \sigma^{2}}}\right]$, donde, como se sabe, debe cumplirse que el área de esta función es la unidad: $\int_{a}^{b} F(x) \cdot d x=1$.

Por otro lado, la media de la FDP es: $\int_{a}^{b} x \cdot F(x) \cdot d x$. Este constituye, de hecho, el valor de la media ponderada, es decir, el valor de la media de la OCDE (Cuadro 1). La varianza en cambio es: $\int_{a}^{b} x^{2} \cdot F(x) \cdot d x-\left[\int_{a}^{b} x \cdot F(x) \cdot d x\right]^{2} ; \mathrm{y}$, por último, el coeficiente de variación: $\mathrm{CV}=\frac{\sqrt{\int_{a}^{b} x^{2} \cdot F(x) \cdot d x-\left[\int_{a}^{b} x \cdot F(x) \cdot d x\right]^{2}}}{\int_{a}^{b} x \cdot F(x) \cdot d x}$ 